\title{
Acoustical Sensing of Small-Scale Vertical STRUCTURES IN ZOOPLANKTON ASSEMBLAGES
}

\author{
By D.V. Holliday, R.E. Pieper, \\ C.F. Greenlaw and J.K. Dawson
}

$\mathrm{O}$ BSERVATION OF AQUATIC animals in their natural environments remains a major challenge in both biological oceanography and limnology. Critical processes in feeding, reproduction, growth, and predation in small zooplankton occur at scales from fractions of millimeters up to scales that match the ambits of individuals. It is often difficult to reproduce all essential features of the marine environment in the lab, where observation of smallscale processes is more tractable than at sea. Therefore there is continuing interest in improving our ability to observe and quantify in situ spatial and temporal changes in the distribution and abundance of zooplankton in relation to natural physical, chemical, and other biological fields.

Making accurate measurements of spatial distributions is difficult. Conventional CTD packages, fluorometers, and acoustical sensors, when used in a "cast" mode from a ship, tend to have $\sim 1 \mathrm{~m}$ vertical resolutions. The time it takes to make a measurement in relation to the "drop" rate, the necessity to pump water through some kinds of sensors, the need to acquire and average multiple samples in a quasi-random, statistically nonstationary local environment, and the coupling of the motions of the ship to the sensor all contribute to "smearing" spatial pattern in the data. These practical issues tend to reduce the spatial resolution of measurements one obtains at sea.

In many of our acoustical field studies of plankton, sea conditions and consequent uneven sampling in depth caused us, in

D.V. Holliday and C.F. Greenlaw, Tracor Aerospace Analysis and Applied Research Division, 4669 Murphy Canyon Road, San Diego, CA 92123, USA. R.E. Pieper and J.K. Dawson. University of Southern California, Hancock Institute for Marine Studies, 820 South Seaside Avenue. Terminal Island, CA 90731, USA. post-acquisition processing, to integrate over $2 \mathrm{~m}$ depth intervals even though the data were collected at submeter resolutions. Two meters was an empirically determined compromise between achieving as much vertical resolution as possible. while averaging enough independent measurements of scattering to obtain good estimates of the acoustical scattering power spectrum, thereby improving our estimates of abundances and sizes of zooplankters present in a given depth interval. Averaging data into $2 \mathrm{~m}$ depth bins has succeeded in revealing a variety of patterns and correlations between ocean physics, phytoplankton, and zooplankton distributions.

Such averaging can, however, lead to an underestimate of the intensity of structure smaller in extent than the bin interval. For example, a $10 \mathrm{~cm}$ thick layer of small zooplankton will appear in a $2 \mathrm{~m}$ average to have a peak abundance 20 times less than is actually the case, even though the total biomass reported for the interval is correct. The differences between actual distributions and apparent distributions can be ecologically important in many ways (feeding, finding a mate, etc.). Meter scale data are useful in answering some important kinds of process and distributional questions, but we should recognize that even meter scale resolution is still not adequate to allow us to quantitatively visualize the environment from the point of view of millimeter-sized organisms.

\section{Small-Scale Scattering Structures in a Shallow Fjord}

In our first example we describe small-scale vertical structure in marine zooplankton in a small fjord, West Sound, at Orcas Island in Washington State's San Juan Islands (Fig. 1). The upper end of this sound is relatively shallow, the bottom is soft mud, and the en- tire basin is subject to relatively strong tidal forcing. Data discussed here were collected in July 1995.

We used a shore-powered, bottommounted, inverted (surface-facing) echo sounder to measure volume scattering strength (Sv) profiles at four frequencies $(265,420,1,100$, and $3,000 \mathrm{kHz})$ specifically selected to be sensitive to the presence of small zooplankton. Every $2 \mathrm{~min}$, 24 (interleaved) scattering intensity profiles were collected at each frequency, range binned in $0.5 \mathrm{~m}$ intervals from 0.75 $\mathrm{m}$ above the bottom to $21.75 \mathrm{~m}$ from the transducer face (i.e., just below the surface). Ensemble averages of the 24 echoranging cycles were made, and absolute volume scattering strength profiles were computed by accounting for spreading, absorption, and various system parameters. Ensemble averages of the volume scattering strength profiles, pressure (tide), and temperature at the base of the acoustical sensor were transmitted to a shore site for real-time display and storage. Power was supplied from the shore site.

Two hours of data from this mooring will be examined. Tidal changes in water depth were measured with a pressure sensor on the up-looking sounder (Fig. 2a). Acoustical volume scattering strength profiles from the $265 \mathrm{kHz}$ and $1.1 \mathrm{MHz}$ sounders are illustrated in Figure 2, b and c. A thin sound-scattering layer can be seen in the $265 \mathrm{kHz}$ record (Fig. 2b), at $\sim 17$ m near the start of the record. Note that height above the bottom is used rather than depth below the changing surface. When initially detected, the layer thickness was $\leq \frac{1}{2} \mathrm{~m}$. The peak intensity (averaged over the system's half-meter range resolution) is probably underestimated. As time passed, the thickness of the layer increased to slightly more than 1 $\mathrm{m}$, diffusing into a thicker, more complex structure at the time of high tide (near the 


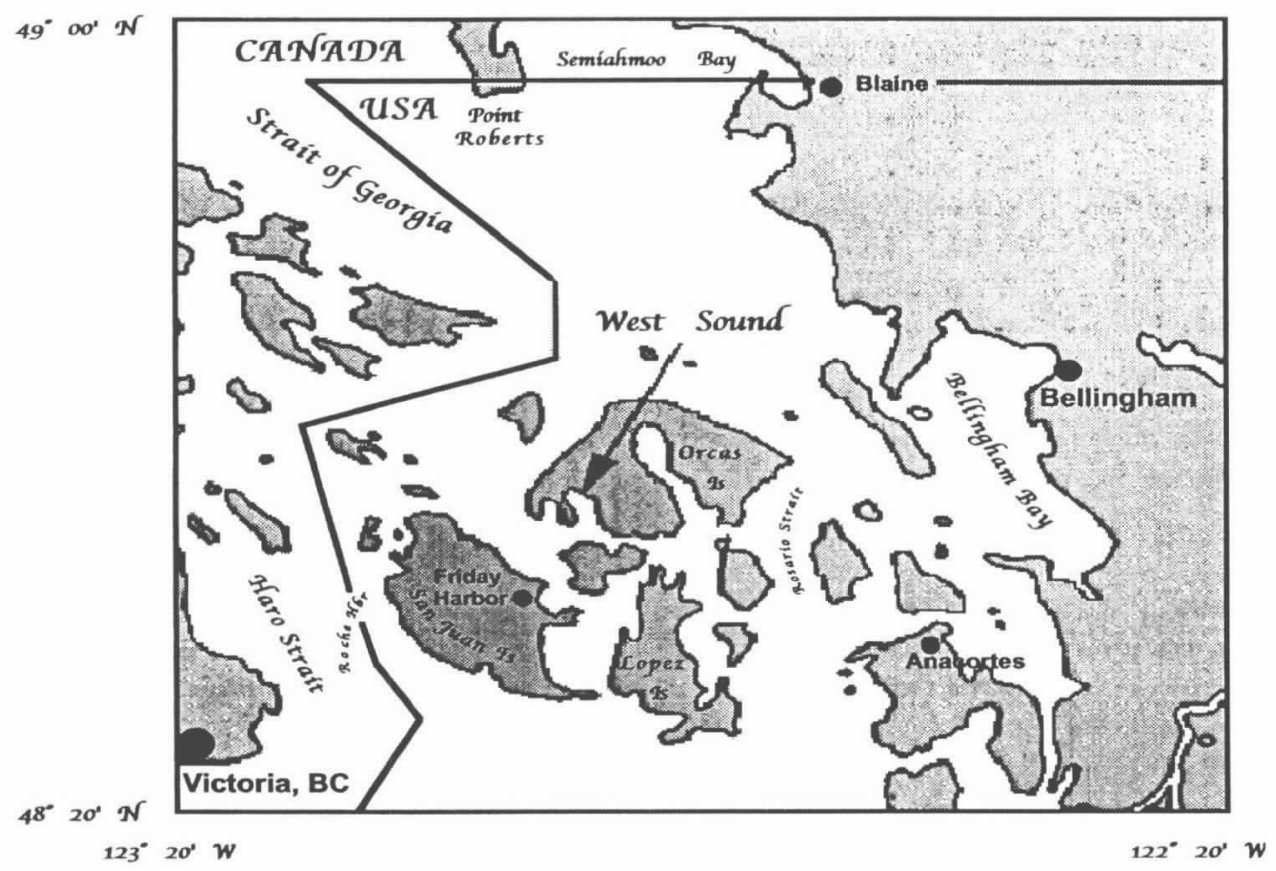

Fig. 1: A small, shallow fjord, West Sound is located in the San Juan Islands, WA, in the extreme northwestern United States.

end of the record). At $265 \mathrm{kHz}$, volume scattering strengths in the layer varied between -60 and $-49 \mathrm{~dB}$. The $420 \mathrm{kHz}$ record (not shown) and the $1.1 \mathrm{MHz}$ record (Fig. 2c) reveal similar patterns for the thin layer, with scattering at levels between -55 and $-47 \mathrm{~dB}$ at $420 \mathrm{kHz}$ and between -50 and $-35 \mathrm{~dB}$ at $1.1 \mathrm{MHz}$.

At 1.1 MHz (Fig. 2c), sound scatterers not visible at $265 \mathrm{kHz}$ (Fig. 2b) were observed as they passed over the sounder. A thin, variable intensity layer of high level scattering, limited to a couple of meters from the bottom, appears to dissipate near the end of the record (i.e., near slack water). In addition, short episodes of high scattering levels appeared early in the 1.1 $\mathrm{MHz}$ record, beginning in mid-column and extending to the surface. Excluding the episodes of high scattering at the beginning of the record, scattering levels just under the surface of the water were both higher and extended deeper into the water column at the end of the record than at the beginning. The wind was negligible, the area was sheltered from surface waves originating elsewhere, and there was no whitecapping or even breaking waves at the shoreline. The most likely explanations for variations in the observed scattering involve changes in the distribution of living organisms above the sounder.

In this work the sensor was deployed as an ancillary measurement tool for an acoustics program, thus the principal inter- est was in describing variability in levels of acoustical scattering rather than in defining the underlying biological causes of the scattering. There was no opportunity to collect samples of zooplankton or fish, quantitative data on tidal velocities, velocity profiles, or CTD information. A diver participant in the project reported the following: "We would often pass through a surface layer, about a meter thick, of plankton on our dives. Zooplankton seemed to be mostly crustaceans mixed in with the phytoplankton. It often was so dense that the critters on our suits were noticeable after exiting the water" (L. Self, personal communication). Quantitative physical, optical, and acoustical measurements in East Sound, nearby on the same small island, have revealed a complex, highly stratified, multilayer, tidally driven water column (e.g., see other papers in this issue). Measurements with an acoustic doppler profiler current (ADCP) have revealed that multiple layers of the water column in East Sound are moving in different directions with attendant shear and turbulence at the layer interfaces (P. Donaghay, personal communication). We have no reason to believe that West Sound is any less complex.

Extensive side scan sonar work at 50, 100 , and $500 \mathrm{kHz}$ was conducted near this mooring, but only a few individual fish were observed, and fish school-like contacts were not observed during the pe- riod illustrated. Likewise, divers reported that the abundance of medusae and ctenophores was low. Our visual observations also suggest that the numbers of medusae and ctenophores in the upper few meters at this site were very low during this data collection period.

At these high frequencies, the absorption of sound in seawater is very large (at 3 $\mathrm{MHz}, \sim 2.4 \mathrm{~dB} / \mathrm{m})$. At ranges of more than $\sim 5 \mathrm{~m}$ above the sensor, reverberation-tonoise ratios for the $3 \mathrm{MHz}$ sounder would not support an inverse calculation of the type we prefer to use, i.e., the multifrequency non-negative least squares (NNLS) method, to estimate size spectra and abundance (Holliday and Pieper, 1995). To obtain information about the size and abundances of the scatterers, a two-frequency inverse algorithm (Greenlaw, 1979; Mitson et al., 1996) was employed. Ratios of volume scattering strengths were calculated by taking the difference between the (logarithmic) volume scattering strengths measured for $265 \mathrm{kHz}$ and $1.1 \mathrm{MHz}$ (Fig. 2d). Greenlaw (1979) pointed out that the size of the scatterer that dominates the acoustical scattering is embedded in these ratios of scattered intensity (differences in Sv). Given a few assumptions about, or $a$ priori knowledge of the scatterers, then for several common marine scatterers, mathematical models exist that will allow estimation of the size and abundance of the dominant scatterer. The interested reader can find a summary of these assumptions in Holliday and Pieper (1995). Based on the frequency dependencies we observed for this layer, the diver's observations, and our experience in the fjords of the San Juan Islands, it appears that (with a few notable exceptions) small crustaceans were the dominant cause of the scattering shown in Figure 2. Although models are available for several generalized zooplankton taxonomies (copepod-like, euphausiid-like, bubble-bearing fish larvae, salp-like, etc.), most zooplankton assemblages are a mix of genera and organisms with a variety of physical shapes. When measurements at sufficient number of frequencies are available, one can sometimes successfully estimate abundance in several (shape-defined) taxonomic classes, by size within the class (Holliday, 1977). When one must work with a limited number of frequencies, it is best to choose the simplest single model that describes the shape (genera) thought, or known from direct sampling, to contribute the most energy to the acoustical scattering. For an assemblage dominated by crustaceans similar 
Tide (water column height)
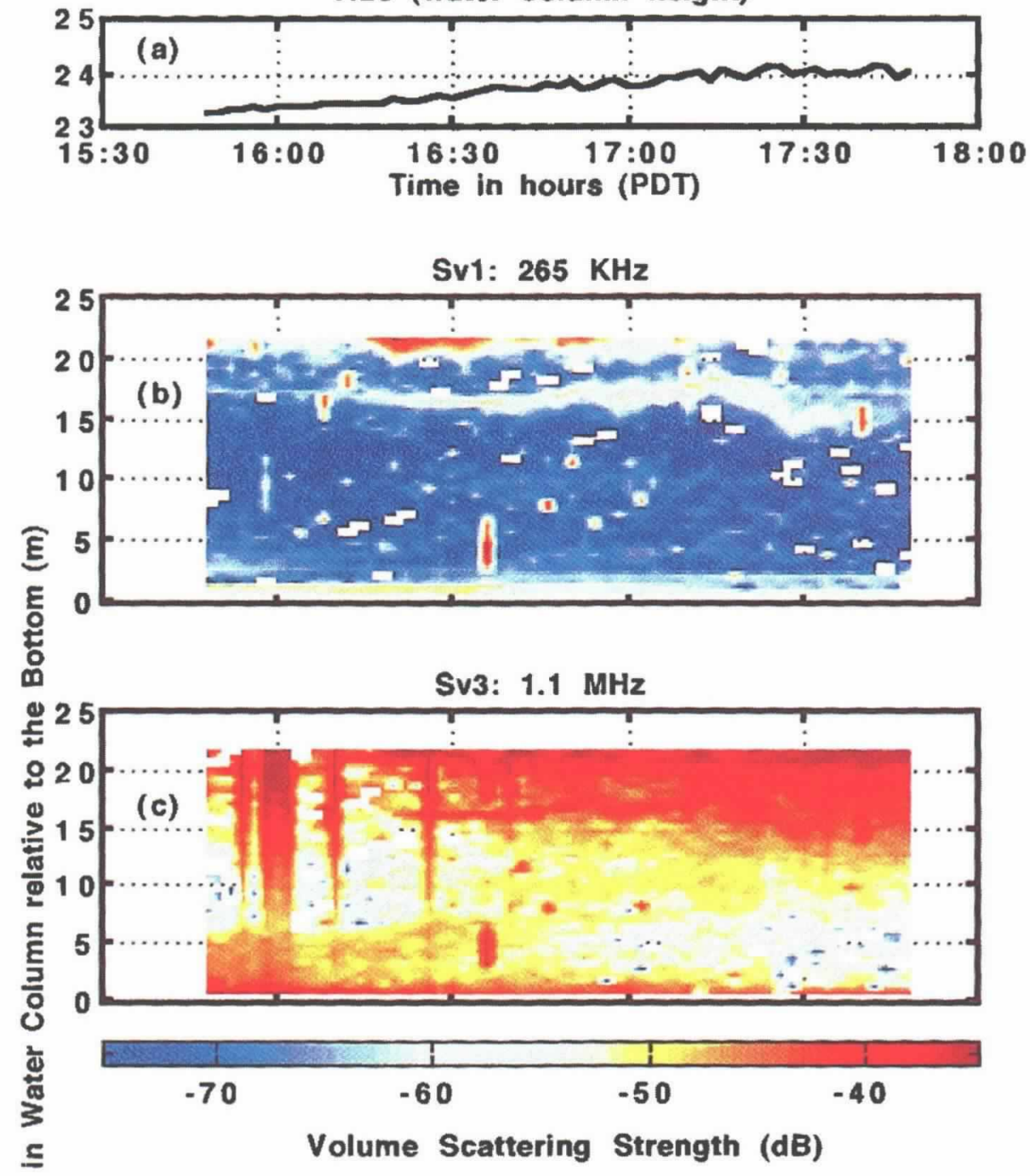

\section{Sv3 - Sv1}

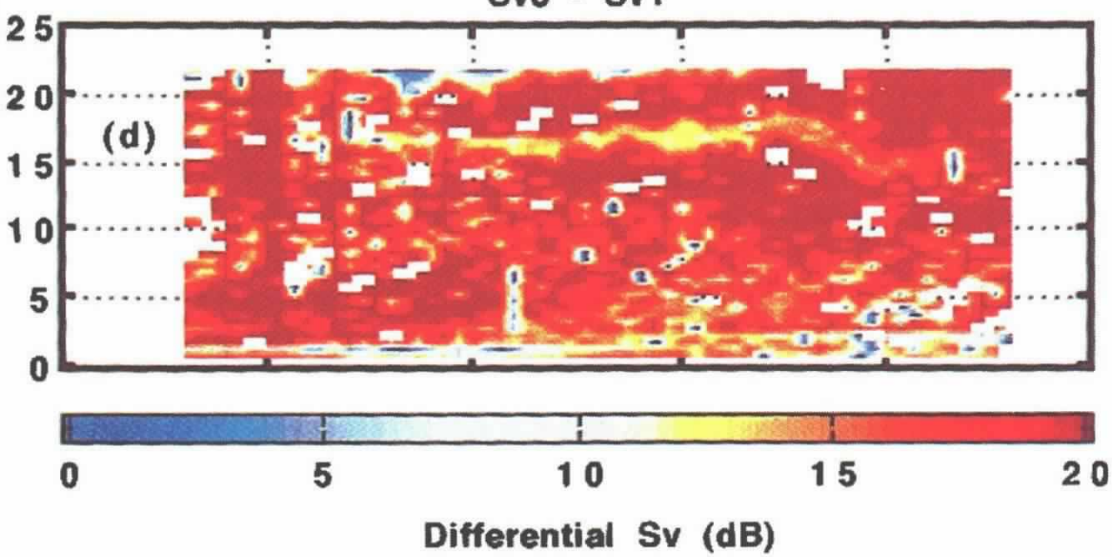

Fig. 2: Two hours of tide level data (a) are illustrated for a station in West Sound, WA, during July 1995. Acoustical scattering data, collected at 2 min intervals and in $0.25 \mathrm{~m}$ vertical bins are also displayed for the $265 \mathrm{kHz}(\boldsymbol{b})$ and $1.1 \mathrm{MHz}$ (c) channels of a four-frequency bottom mounted, up-looking echo sounder. The color legend under the $1.1 \mathrm{MHz}$ data (b) also applies to the $265 \mathrm{kHz}$ display (c). The sizes of the dominant scatterers, in this case mostly small crustaceans, are embedded in the difference of the logarithms of the measured volume scattering (ratios of the intensities) as illustrated in $\boldsymbol{d}$.

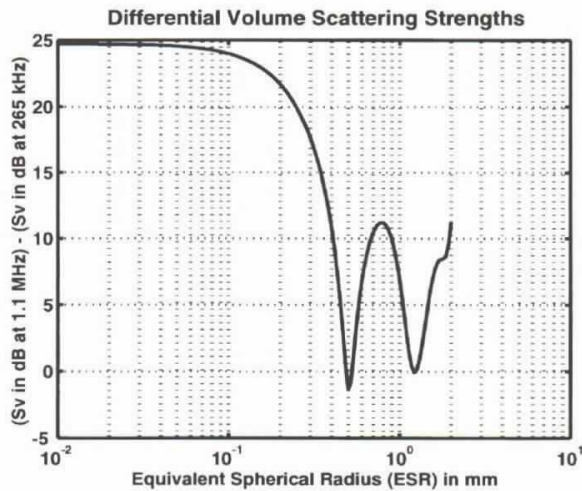

Fig. 3: The dependence of the difference of volume scattering strengths, measured in decibels (log scattering intensities), on the size of the dominant acoustical scatterers in the water column is illustrated for scatterers that follow a truncated fluid sphere scattering law.

to a calanoid copepod (roughly an ellipsoid of revolution), this is the truncated fluid sphere model (Pieper and Holliday, 1984), and this is the scattering descriptor chosen for the present analysis. The relation between the ratio of volume scattering strengths (Svs) at $265 \mathrm{kHz}$ and $1.1 \mathrm{MHz}$, expressed as differential Svs versus scatterer size (ESRs) is illustrated in Figure 3. The equivalent spherical radius (ESR) is the radius of a sphere that would contain the same volume as the scatterer displaces. This model must be used with some care, because unlike the high-pass model originally used by Greenlaw, it is not monotonic with the product of scatterer size (ESR) and the wavenumber $(\mathrm{k})$. One result of this nonlinear, nonmonotonic form is that there is no closed form analytical solution, therefore one must rely on numerical techniques. Another result of this functional form is the possibility that there will be multiple solutions for size for some ratios of Svs. Using different combinations of the available frequencies often allows one to resolve ambiguities in the dominant size calculated by this method.

The biomass predicted by the two-frequency algorithm (Fig. 4a) and the calculated size of the dominant scatterer (Fig. 4b) reveal that the thin layer at $\sim 17 \mathrm{~m}$ consisted principally of animals with an equivalent spherical radius of $\sim 0.3 \mathrm{~mm}$. The ratio of the Svs at $420 \mathrm{kHz}$ and 1.1 $\mathrm{MHz}$ also produced dominant size and biomass estimates that are consistent with the estimates for the layer in question, as illustrated in Figure 4. If the animals had shapes similar to calanoid copepods, this would translate to overall lengths of be- 


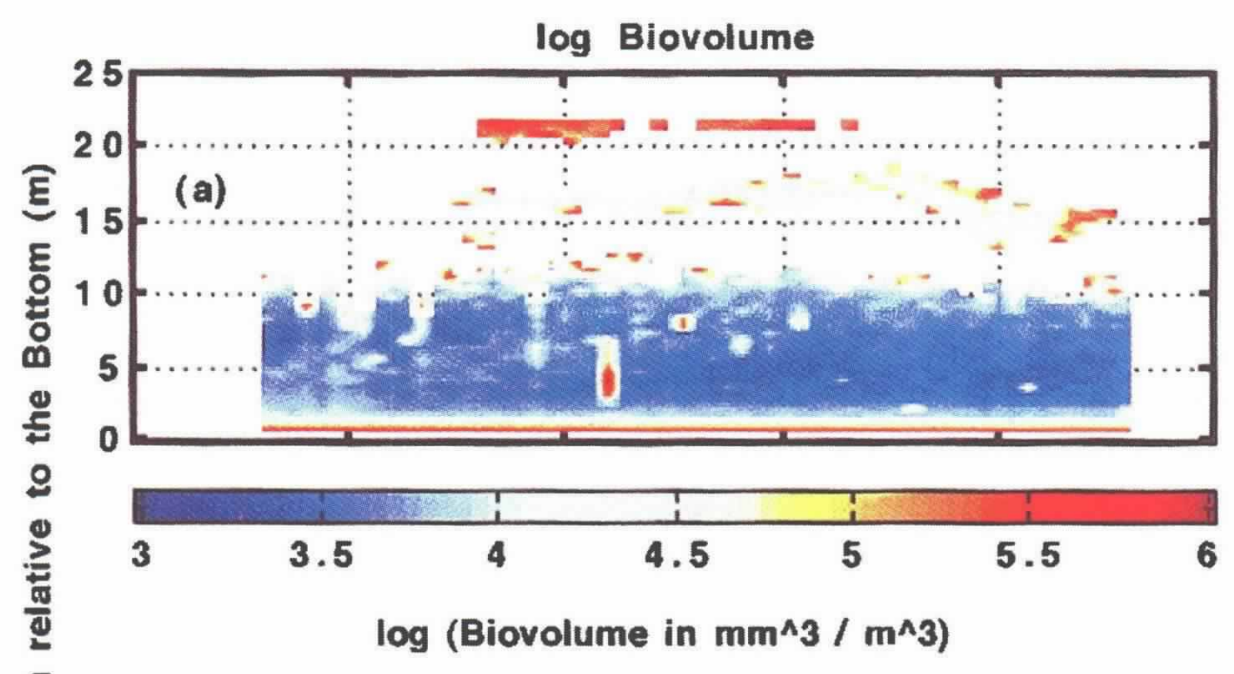

동

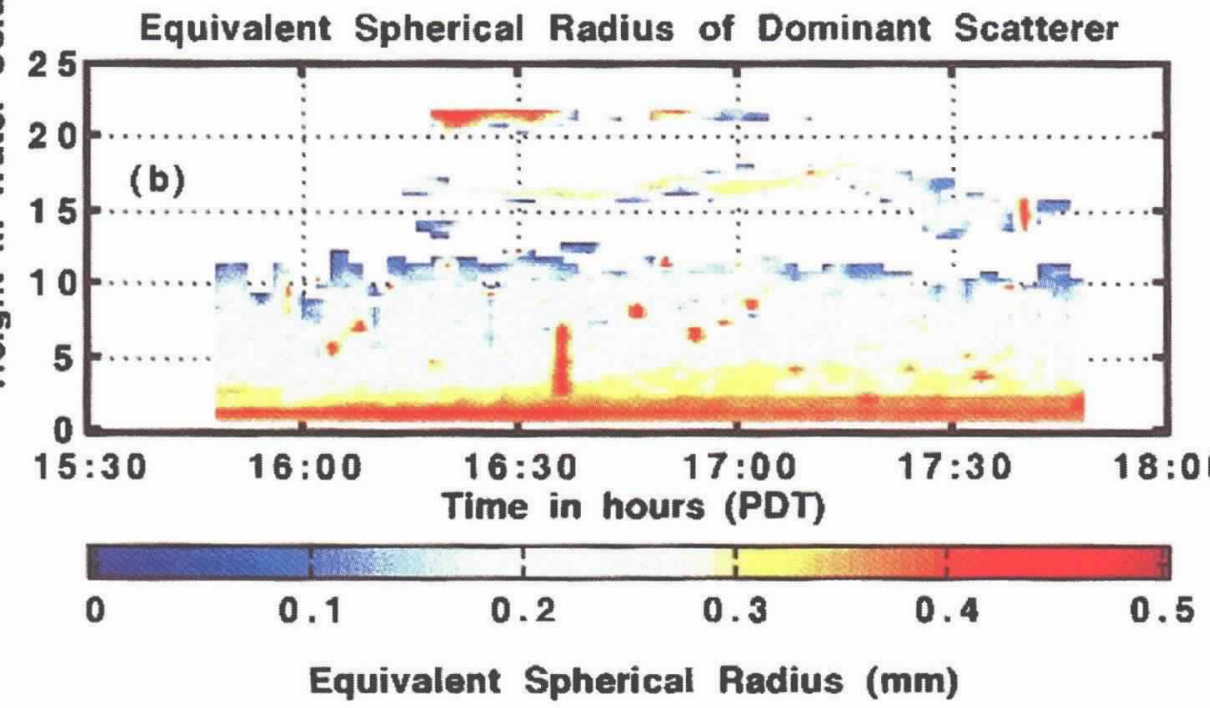

Fig. 4: Estimates of biovolume and zooplankton size are illustrated for the acoustical data displayed in Fig. 1. Blank pixels are displayed when the scattering was too low to result in a valid estimate; when the scattering ratios indicated that the truncated sphere model used for scattering from individuals was not correct; and when the scatterer size was larger than could be uniquely determined with the two-frequency inverse technique used. Examination of additional data at $420 \mathrm{kHz}$ (not displayed here) revealed that most of the blank pixels in this data set were due to low numbers of small zooplankters.

tween 1.2 and $1.5 \mathrm{~mm}$. Based on this size, the biovolume, a measure of biomass that is analogous to displacement volume, within the layer ranged between $\sim 15,800$ and $39,700 \mathrm{~mm}^{3} / \mathrm{m}^{3}$. This would correspond to a range of nominal dry weights, assuming that the layer is mostly small crustaceans, from 3,000 to 10,000 $\mathrm{mg} \mathrm{C} / \mathrm{m}^{3}$. Although this is a dense layer, it is not unusual. Much higher concentrations of copepods of similar sizes have been observed in even thicker layers (e.g., Alldredge et al., 1984). The authors have often observed similar layers in the coastal waters of Southern California (Holliday and Pieper, 1980; Pieper and
Holliday, 1984), on the shelf off Cape Canaveral, FL (Holliday et al., 1989), and in the Irish Sea (Holliday, 1993).

In addition to the thin, dense layer near $18 \mathrm{~m}$, and the thin, sparsely populated layer of slightly larger animals (0.38-0.45 mm ESR) within a few meters of the bottom, there appears to be a boundary in the vicinity of $10 \mathrm{~m}$ (height off the bottom) that divides the water column. Above this boundary, scattering levels at $265 \mathrm{kHz}$ below and above the thin midwater layer were too low to allow us to make valid size estimates with the two-frequency algorithm. In the absence of a valid estimate of the size of the scatterer, the two-frequency algorithm returns no data for biomass. This is the reason for the absence of data in parts of the upper half of the water column in Figure 4, a and b. Small (0.12-0.2 mm ESR) plankton appear to dominate the scattering in the lower half of the water column. The biomass is diffuse but with a heterogeneous or "patchy" distribution in both abundance and size. Very near the surface, relatively high abundances of larger $(\sim 0.4-0.5 \mathrm{~mm}$ ESR) organisms were present for nearly $1 \mathrm{~h}$ in the middle of the period illustrated. There is a suggestion in the data that several high biomass concentrations or "patches" of scatterers are associated with the top of the apparent bioboundary near $10 \mathrm{~m}$. Small, high biomass patches also appear to be associated with the thin midwater layer.

\section{Plankton Layers Near the Shelf-Slope Break}

Over several years we have collected numerous vertical profiles of acoustical volume scattering strength at multiple frequencies. One of our objectives was to quantify these relationships in order to gain an ability to "invert" the acoustical data, thereby estimating zooplankton biovolume spectra by size with depth (Costello et al., 1989; Holliday and Pieper, 1995). While pursuing the relationship between the distribution, size and abundance of plankton, and the acoustical scattering, we have often observed profiles similar to those displayed in Figure 5. These data were collected on a narrow, shallow shelf at the north edge of the San Pedro basin near Long Beach, CA, in June 1996. The profile was made in the late afternoon, starting at 19:41 PDT. Temperature is displayed in Figure 5a, and Figure 5b is the fluorescence profile. The volume scattering spectrum is shown in Figure 5c and the biovolume-size spectrum is given in Figure 5d. The profile of total biovolume, summed over all the sizes displayed in Figure 5d, is provided in Figure 5e. The multifrequency inverse method described in Holliday and Pieper (1995) was used to map volume scattering into the plankton biovolume size-abundance profile.

Abnormally calm sea surface conditions were present during this particular cast, and we took advantage of the weather and low swell to collect a relatively high resolution profile, lowering our Tracor Acoustical Profiling System (TAPS) six-frequency sonar system at a rate of $<0.04 \mathrm{~m} / \mathrm{s}$. A complete set of acoustical data are collected along with 

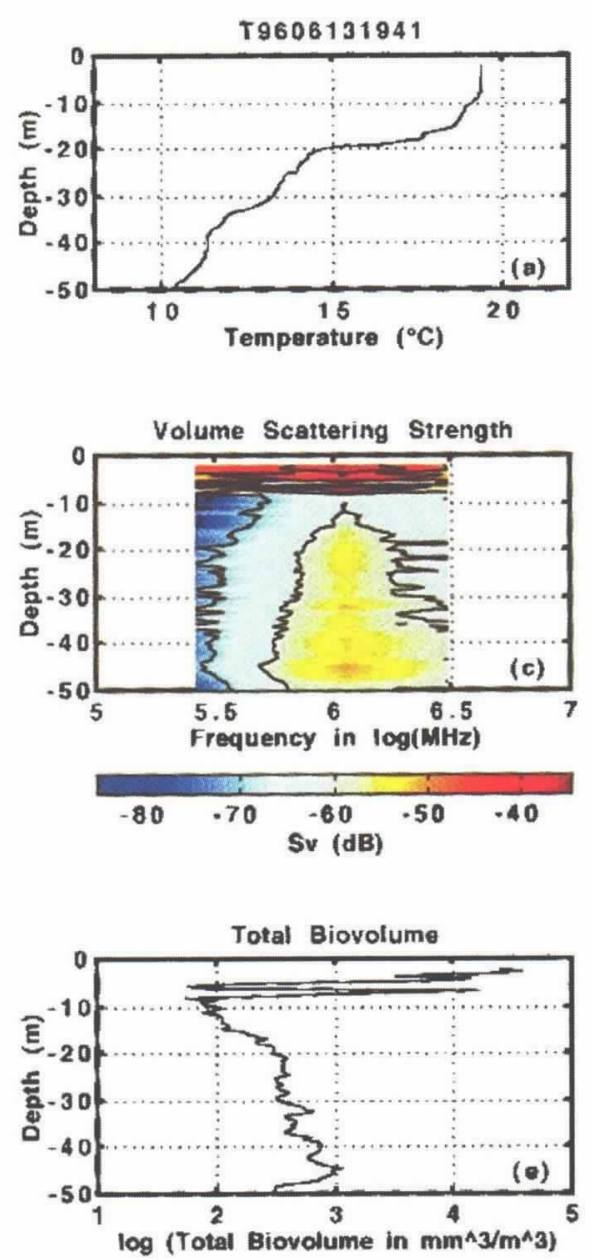

Fig. 5: Temperature (a), fluorescence (b), and volume scattering strength spectrum profiles (c) are illustrated for a cast $\sim 15 \mathrm{~km}$ south of Long Beach, CA, in June 1996. The calculated biomass-size spectrum profile $(\boldsymbol{d})$ and the total biovolume profile (e) reveal that much of the biomass at this station was concentrated in three very thin layers in the upper mixed layer.

temperature by this system every $4 \mathrm{~s}$. The volume scattering strengths were averaged into vertical bins of $\sim 0.25 \mathrm{~m}$.

Structure can be observed on scales from that of the basic resolution of the acoustical sensor, $\sim 0.25 \mathrm{~m}$ in this instrument configuration, to scales of $>100 \mathrm{~m}$, the depths of the usual casts made with the instrument. The dominant structures in this particular cast were the three thin layers in the upper mixed layer. The water depth at this station was $53 \mathrm{~m}$. The shallowest layer, centered at $\sim 3.25 \mathrm{~m}$, contained $63.5 \%$ of the water column biomass. A second layer, with a thickness of $<0.5 \mathrm{~m}$ was centered at $4.25 \mathrm{~m}$ and contained $6.5 \%$ of the biomass. The third layer, between 6.5 and $7.5 \mathrm{~m}$, represented another $8.4 \%$ of the biomass. The total water column plankton biovolume was $31,208 \mathrm{~mm}^{3} / \mathrm{m}^{2}$. Early evening MOCNESS (Multiple Opening Closing Net En-
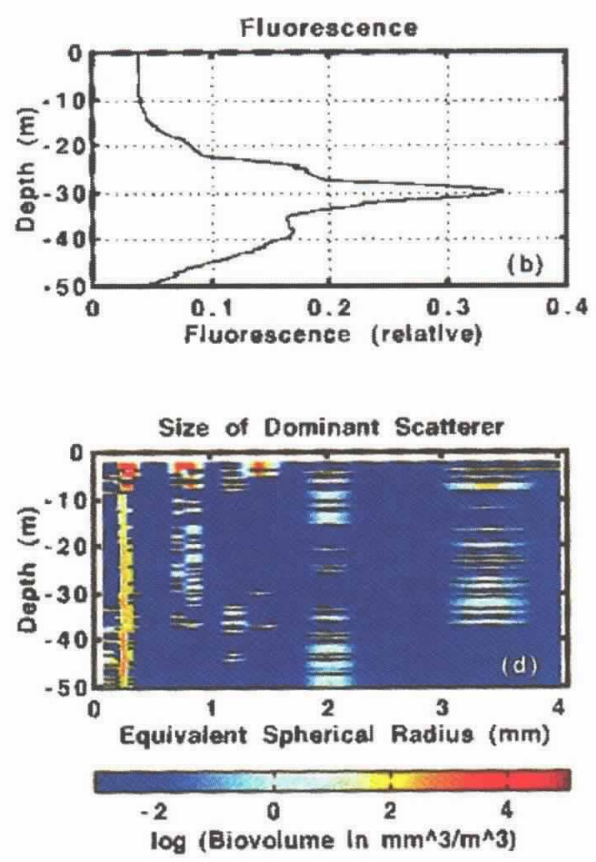

phytoplankton, based on fluorescence measurements, and in the zooplankton, from multifrequency acoustical measurements, can have horizontal extents of tens of kilometers (Holliday et al., 1989; Holliday, 1993). These small-scale temperature structures are also often, but not always, associated with fluorescence maxima and with structure in profiles of optical scattering and optical transmission (Donaghay et al., 1992; Cowles and Desiderio, 1993). We use the term associated, rather than correlated, for several reasons. In our observations, the acoustical estimates of zooplankton biomass (biovolume) distributions sometimes have shapes similar to the temperature gradient at such an anomaly, with the peaks of the zooplankton distributions and the maximum of the temperature gradient coinciding in depth. Both temperature gradients and small-scale turbulence can cause acoustical scattering by virtue of impedance discontinuities and variations in the sound speed of the reflected sound waves, but in our data both the levels and the spectrum of the scattering seem to best fit a model that associates peaks in zooplankton biomass with these structures. We have also observed that the strength of the acoustical scattering does not appear to be correlated with the magnitude of the gradient in the pycnocline. Often, these small steps or variations in temperature occur at boundaries of the zooplankton distribution, delimiting lower or upper bounds of particular plankton sizes or assemblages of sizes. Finally, we are cautious about making statements about correlation and coherence because the acoustical tools at hand, such as the TAPS, are working near the limit of their spatial resolution to define these structures. Nonetheless, it is clear that there are processes occurring at submeter scales that are reflected in the response of both phytoplankton and zooplankton to the physical environment.

\section{Summary and Conclusions}

In the illustrations we have provided, a substantial amount of the zooplankton biomass was concentrated in heterogeneous, small-scale structures, one form of which is a very thin layer. The biological and physical reasons for this distribution are poorly understood, but it is clear that this kind of distribution is neither unusual, nor rare, in coastal regions. This kind of distribution clearly has implications for predation by larger animals, e.g., for larval and juvenile fish. Its causes may be rooted in distributions of food, 
i.e., phytoplankton, but may also be at least in part physically driven (e.g., the advantages of moderate levels of turbulence are discussed by Rothschild and Osborn 1988). To appropriately observe and understand this structure and the plankton's response, we not only require acoustical sensors with at least an order of magnitude better spatial resolution than are currently available (i.e., $\sim 10$ $\mathrm{cm}$ ), but we also require a new protocol for deploying the sensors, one that is decoupled from the motion of a ship. Bottom-mounted systems are one solution for shallow water environments, especially if used in conjunction with ADCPs. For deeper environments, where sound absorption requires that acoustical sensors be placed near the organisms of interest, buoyancy-driven and free-fall profilers and profiling winches are attractive alternative platforms. Simply defining the distribution of the plankters at small scales, without describing their environment at similar scales is not sufficient.

For about a decade, oceanographers have been working to develop advanced sensors that can be used to examine aquatic environments faster, in more detail, and with improved spatial and temporal resolution. Some of the technologies finding applications in this quest include high-resolution imaging optics, video plankton recorders, high-frequency acoustical zooplankton sensors, optical fiber fluorometers, a variety of turbulence sensors, faster thermistors for measuring temperature, optical particle counters, multispectral optical transmissometers, and holographic cameras. Used alone, each of these technologies provides a view of only a part of the components of an ecosystem. Many ocean scientists believe measurement of fine- and microscale struc- tures needed to describe submeter scale distribution and behavior of the animals, plants and their physical environment and their $\mathrm{dy}$ namic interactions ultimately will require a quantitative integration of several technologies. Individually, some of these sensor technologies have matured, and they are routinely used to examine animals and their environments on meter and submeter scales. Together, different technologies can be used either to measure different properties of the same animals in a small volume, or when they have substantially different spatial resolutions, they can be "nested," with the lowresolution system setting the spatial context for the high-resolution sensor (e.g., an optical system with a resolution of a few cubic millimeters can examine a subset of a liter of water being observed with an appropriate acoustical system). It appears to be timely to begin working on this quantitative synthesis of technologies in the hope that the result will be synergistic. This, in our opinion, although requiring a dedicated effort by multiple investigators, is one of the more promising avenues for research and development in sensors for studying plankton and its environment for the next decade.

\section{Acknowledgements}

This research was sponsored by the Office of Naval Research and by the National Science Foundation. We would like to acknowledge the invaluable assistance of R. Player, L. Howell and P. Jarrett in the conduct of the work.

\section{References}

Alldredge. A.L., B.H. Robison. A. Fleminger, J.J. Torres, J.M. King and W.M. Hamner. 1984: Direct sampling and in situ observation of a persistent copepod aggregation in the mesopelagic zone of the Santa Barbara Basin. Mar. Biol, 80. 75-81.
Costello, J.H., R.E. Pieper and D.V. Holliday, 1989: Comparison of acoustic and pump sampling techniques for the analysis of rooplankion distributions. J. Plankton Res. 11, 703-7099.

Cowles, T.J. and R.A. Desiderio, 1993: Resolution of biological microstructure through in situ fluorescence emission spectra. Oceanography 6. 105-111.

Donaghay, P.L.. H.M. Rines and J.M. Sicburth, 1992: Simultaneous sampling of tine scale biological, chemical, and physical structure in stratified waters. Arch. Hydrobiol. Beth. Ergebn. Limnol. 36, 97-108.

Greenlaw, C.F., 1979: Acoustical estimation of zooplankton populations. Limnol. Oceanogr. 24. 226-242.

Holliday, D.V., 1977: Extracting bio-physical information from the acoustic signatures of marine organisms. In: Occanic Sound Scattering Prediction, N.R. Anderson and B.J. Zahuranec, eds. Plenum Press. Marine Science Series, New York, Vol. 5, 619-624.

1993: Applications of advanced acoustic technology in large marine ecosystem studies. In: Large Marine Ecosystems: Stress. Mitigation, and Sustainability, K. Sherman, L. Alexander and B. Gold, cds. AAAS Press, Washington, DC, Publication 92-39S, 301-319.

and R.E. Pieper, 1980: Volume scattering strengths and zooplankton distributions at acoustic frequencies between 0.5 and 3 MHz. J. Acoust. Soc. Am. 67. 135-146. and R.E. Pieper. 1995: Bioacoustical oceanography at high frequencies. ICES J. Mar. Sci. 52, 279-296.

R.E. Pieper and G.S. Kleppel, 1989: Determination of zooplankton size and distribution with multifrequency acoustic technology. J. Cons. Int. Explor. Mer, t6, 52-61.

Mitson. R.B., Y. Simard and C. Goss, 1996: Use of a two-frequency algorithm to determine size and abundance of plankton in three widely spaced locations. ICES J. Mar. Sci. 53, 209215.

Pieper, R.E. and D.V. Holliday, 1984: Acoustic measurements of zooplankton distributions in the sea. J. Cons. Int. Explor. Mer. 4I, 226-238.

Rothschild, B.J. and T.R. Osborn, 1988: Small-scale turbulence and planktonic contact rates. $J$. Plankton Res. 10,465-474. 